\section{Somatotopic map of the flying fox}

SiR - In the interesting article by Calford et al. on the unusual somatotopic cortical map in the grey-headed flying fox, Pteropus poliocephalus, and also in the comment by Jones ${ }^{2}$ on the article, one point escaped emphasis: the caudal shift of the somatotopic representation of the distal forelimb has taken place by reflection from rostral to caudal (that is the first digit or thumb remains lateral and adjacent to the face), instead of by rotation from rostral to caudal (in which event the thumb would lie medial and the fifth digit would be located adjacent to the face). In contrast to the reflection of the representation of the forelimb, the representation of the hindlimb appears to have rotated through about 90 degrees, so that it points medially (instead of rostrally as in most mammals). Whether this difference in the shift of the fore- and hindlimbs is related to their different functions (flying and feeding in the case of the forelimb, landing from flight in the case of the hindlimb) remains an open question.

Jones $^{2}$ has addressed a related issue: what is the advantage of representing the body surface on flat cortical maps instead of as a bolus of cells? Rostral/caudal and medial/lateral appear to be the spatial relationships in the periphery that need to be maintained in the cerebral cortex; dorsal/ventral seems a less important direction, being coded sometimes as caudal/ rostral (for example in the axial/distal parts of a limb in quadrupes) and sometimes as lateral/medial (for example in the position of the head relative to the limbs and trunk). If, then, one direction in the periphery does not have to be maintained in the cortex, a flat map can achieve all that a bolus would achieve, with the advantage of much less complex connections between thalamus and cortical surface.

G. ETtLinger

Department of Psychology,

University of Bielefeld, 4800 Bielefeld I, FRG

1. Calford, M.B., Graydon, M.L., Huera, M.F., Kaas, J.H. \& Pettigrew, J.D. Nature 313, 477-479 (1985).

2. Jones, E. Nature 313, 434 (1985).

\section{Spatial relationship and extrafoveal vision}

SIR - A recent letter by Rentschler and Treutwein ${ }^{1}$ describes a qualitative difference in human visual acuity between central foveal vision and a region $2^{\circ}$ from the fovea when the task is to discriminate between stripe patterns that differ in appearance but are mathematically similar in everything but phase relationship between first and third spatial harmonics. For the pairs that are easily discriminated both foveally and at $2^{\circ}$, the appearance of the stripes differs in the contrast of a narrow dark band on a lighter grey stripe. In the patterns that can be discriminated foveally and at $2^{\circ}$, the darker bands in each discrimination task are identical in quality and differ only in that their position with respect to the lighter bands is mirror-image reversed in the two images.

Rentschler and Treutwein conclude that (1) there is a qualitative difference between foveal and nonfoveal vision for spatial localization tasks but not for contrast detection tasks, and that (2) because of this difference between foveal and nonfoveal vision for one type of task but not the other, there must be two types of visual processing involved. We suggest that there might not be a qualitative difference between foveal and nonfoveal vision but rather a quantitative difference - spatial localization might simply decline faster than contrast detection as a function of eccentricity - and that this difference can be explained at least partly in terms of receptive field size and stimulus resolution in complex cells at various levels in the visual pathway, as a function of eccentricity.

Both the letter itself and the accompanying News and Views article by Watt ${ }^{2}$ are couched in terms of the Fourier-analysis theory of cortical function; curiously no mention is made of known receptive field properties of cortical cells. As an alternative to their interpretation, which assumes that the early stages of vision can be regarded as a number of parallel spatial filters (a view that our receptive field studies do not seem to support), we would propose an interpretation (or perhaps a more physiological translation of their interpretation) in terms of the known properties of complex cortical cells. At a given eccentricity, the territory of visual field over which a complex cell can be activated is usually much larger than that of a simple cell, but the optimum stimulus size is approximately the same. A complex cell responds optimally to lines or slits in a specific orientation, but for an optimal response the line width is also critical. The optimal line evokes a response wherever it is shone over a wide area, even though a line thick enough to cover this entire area evokes no response at all. The optimal line width is roughly the same as the width of the region from which a simple cell can be activated, or the diameter of a retinal ganglion cell receptive field centre (for the same eccentricity), implying a preservation of acuity but loss of the spatial localization conveyed by simple cells.

This relative loss of what may correspond to spatial localization without loss of acuity is seen not only in complex cells in area 17, but even more strikingly in prestriate visual areas. Rovamo and Virsu ${ }^{3}$ have shown that human visual acuity at different eccentricities varies as a linear function of cortical magnification in area 17, and Rentschler and Treutwein therefore use magnification to scale the size of their two test patterns for eccentricity: they compare foveal and nonfoveal discrimination using only this one stimulus size ratio. This may be justified if one assumes that resolu@ 1985 Nature Publishing Group tion (that is, acuity) as a function of eccentricity does not change as one goes further along in the visual pathway; physiological comparisons of areas 17 and 18 (see our paper, this issue ${ }^{4}$ ) support this assumption. But we think that magnification in area 17 might not be an appropriate scaling factor for tasks involving spatial localization. Spatial localization could well depend on the size of complex receptive fields, and that clearly does increase beyond 17 , for example, in 18, V4 and MT, and also increases more rapidly for nonfoveal than for foveal vision.

The difficulty in discriminating mirrorimage patterns is more likely to be related to spatial localization, and consequently tailoring stimulus size to cortical magnification in area 17 may be inappropriate. The mirror-image (spatial localization) discrimination is already poorer than the contrast discrimination in the fovea (Fig. 2 of ref. 1) and falls off more rapidly with eccentricity. This parallels the behaviour of complex cells at more central levels of the visual system than area 17. Thus the finding would seem to suggest not a qualitative difference in handling of form foveally and peripherally, but a difference in rates of deterioration with eccentricity between acuity as commonly defined, and something else, possibly spatial localization.

We further suggest that these results do not necessarily implicate two separate visual processes for contrast discrimination and spatial localization. The fact that the relative loss of spatial information seen physiologically in single cells is paralleled in these psychophysical observations suggests to us that spatial localization and contrast information are probably encoded in the same cells and not by separate processes. Only if psychophysical spatial localization were better than predicted by the physiology of complex cells would one need to invoke a separate process for it.

\section{MARGARET S. LIVINGSTONE} DAVID H. HUBEL

Department of Neurobiology,

Harvard Medical School,

25 Shattuck Street, Boston,

Massachusetts 02115, USA

1. Rentschler, I. \& Treutwein, B. Nature 313, 308-310 (1985). 2. Watt, R.J. Nature 31, 266-267 (1985)

. Rovamo, J. \& Virsu, V. Expl Brain Res. 37, 495-510 (1979).

4. Hubel, D.H. \& Livingstone, M.S. Nature 315, 325-327 (1985).

\section{Lysosomes and prohormone}

\section{activation - correction}

IN the letter from $\mathrm{C}$. N. Hales under this title in Nature of 7 March (Vol. 314, 20) an editorial change altered the sense of the final paragraph which should read:

The sequence information which has emerged to date is consistent with the lysosomal hypothesis, suggesting that it may provide a useful framework for making and interpreting structural comparisons in this area.

The hypothesis referred to is outlined in the second paragraph of the letter and in more detail in the ref 3 and 4 in the list cited. 